\title{
La traducción de la publicidad televisiva: la globalización, ca- talizadora de cambios en la estrategia traductora ${ }^{1}$
}

\author{
Rosa Agost \\ Universitat Jaume I de Castelló
}

Spain has always been a prototypical subtitling countr, and it still is. Thirty years of democracy and openness to Europe and the world have not succeeded in changing the habits of its viewers, once imposed by an iron dictatorship, nor have they succeeded in supplanting the complexity of the dubbing industry with the apparent simplicity of subtitling. And yet, the translation of publicity offers a revealing take on the world of subtitling and today's television landscape.

It is my contention that the globalising strategies of publicity, and audiovisual genre that is a kind of reflection of contemporary society (?), as well as the advent of younger generations, more acustomed to multilingualism, might stimulate change in translation practice in this sector, and that this might in its turn lead to more fundamental (future) changes in the way society consumes audiovisual products in a broader sense.

\section{Introducción: algunas preguntas}

El análisis de un corpus extenso formado por espots televisivos de los últimos diez años y una encuesta llevada a cabo en diferentes segmentos de la población nos darán algunas claves para responder a distintas preguntas acerca de la traducción publicitaria: ¿Qué consideración merecen por parte de la sociedad el doblaje y la subtitulación en la actualidad? ¿Cuáles han sido las estrategias de traducción de los espots televisivos en la última década? ¿Ha habido alguna evolución significativa sobre las modalidades más habituales en la traducción publicitaria? ¿Quién decide la estrategia traductora a seguir? ¿Existe alguna tendencia clara en un futuro próximo sobre la proporción del doblaje y la subtitulación en la publicidad televisiva y en la traducción audiovisual en general? ¿En el caso de vislumbrarse alguna tendencia podría extrapolarse a la traducción audiovisual en general?

\section{Hipótesis de trabajo}

El presente trabajo se centra en un amplio estudio de un corpus de más de 800 espots publicitarios y en una encuesta realizada a 460 individuos de diferentes edades, niveles de estudios y conocimientos de idiomas sobre la recepción de la traducción audiovisual con especial atención a la traducción 
publicitaria. Las hipótesis que han motivado este estudio sirven para intentar comprobar si:

- $\quad$ El doblaje es aún la modalidad más aceptada en la España actual;

- Los espectadores consumen productos audiovisuales doblados por inercia y comodidad;

- $\quad$ La subtitulación comienza a ser más aceptada que antes por el público;

- Los jóvenes tienen un conocimiento mayor de idiomas;

- Los jóvenes se muestran más receptivos que las personas de más edad a los textos audiovisuales multilingües (VOS) en sus diferentes formatos (películas de cine, DVD, programas de televisión, juegos multimedia, etc.);

- Cuanto mayor es el nivel de estudios de la población mayor es la aceptación de las versiones originales y subtituladas;

- A los consumidores les gusta la publicidad;

- Los jóvenes admiten mejor que los mayores la publicidad subtitulada;

- $\quad$ Los jóvenes recuerdan más la publicidad original y la subtitulada;

- Los encuestados consideran que la traducción publicitaria la llevan a cabo profesionales de la traducción frente a profesionales de la publicidad o lingüistas;

- $\quad$ Existe una relación muy estrecha entre el tipo de producto y destinatario de un anuncio, por una parte, y la estrategia de traducción elegida;

- La globalización aumenta la necesidad de diseñar publicidad "apta para todos los públicos" y hay determinados grupos de productos que son más susceptibles de adoptar estrategias de traducción más extranjerizantes (no traducción y subtitulación).

Una última hipótesis que no podrá confirmarse en este trabajo sino a largo plazo, es que el consumo de publicidad subtitulada puede ayudar a favorecer la recepción de la subtitulación de otros géneros televisivos como las películas o los documentales en tanto en cuanto convierte en cotidiana una modalidad considerada elitista hasta hace poco.

\section{La traducción audiovisual, la publicidad y los estudios sobre la tra- ducción}

Los conceptos de globalización, publicidad y traducción están íntimamente relacionados. Esta idea queda manifiesta de forma prístina en Snell-Hornby (2006: 129), cuando habla sobre el globalization turn: "It applies directly to technology and commerce, but also to communication and language, to intercultural discourse, and hence to translation". Acerca de la traducción audiovisual, Mayoral (2005: 6) comenta que 
[...] no es un objeto de estudio inmutable y ya definido sino un objeto de estudio condenado al cambio y a la innovación. Realidades susceptibles de ser incluidas en la misma categoría (la llamemos como la llamemos) que la traducción cinematográfica, televisiva o de vídeo, como son los productos multimedia, los juegos de videoconsola, el subtitulado para sordos, el subtitulado en la enseñanza de lenguas extranjeras o la traducción de páginas WEB, serán ignoradas por los estudiosos universitarios si hacemos un uso dogmático de las definiciones.

Entiendo que la traducción publicitaria se incluye también en este grupo que no ha sido considerado objeto de estudio por parte de nuestra disciplina hasta hace poco tiempo (cf. Séguinot 1994; Guidère 2000; Valdés 2000, 2001, 2004, 2005; De Mooij 2004; Bueno 2000 y 2005; Adab \& Valdés 2004, entre otros). De hecho, Mayoral, hablando sobre las fronteras actuales del ámbito de la traducción audiovisual, comenta que se han incorporado al campo de la TAV nuevas especialidades, "alguna de fuerte empuje, como la adaptación publicitaria" (2005: 7). En este sentido, Munday (2004: 215216) concluye su trabajo sobre los retos y relaciones existentes entre la publicidad y los Estudios sobre la traducción con las siguientes palabras:

I set out to show that until recently translation theory has generally ignored advertising or else included it as an illustration of part of a more general theory of translation. Similarly, marketing and brand management have tended to make little mention of translation. Concepts such as audience, equivalent effect, skopos and crosscultural study are clearly of prime importance in this area. However, the focus of translation has been very much on the conventional, progressive outdated, written text despite advertising being one of the clearest examples of the new multimodal, multimedia world of communication.

La pertinencia de este estudio radica, por una parte, en la relevancia del tema ya que, como señala Rodríguez del Bosque (1997: 116), "hablar hoy en día de publicidad es hablar de televisión". Y, con la presencia de Internet en un alto porcentaje de hogares españoles, añadiría que también la relación entre publicidad e Internet también resulta clave. Por otra parte, respecto a los estudios sobre la traducción publicitaria, considero que este estudio inicial supone una aportación en un campo bastante virgen todavía: los estudios empíricos sobre traducción publicitaria. Hace unos años, Fuentes Luque (2001: 69) comentaba sobre la traducción audiovisual:

Frente al panorama de estudios básicamente teóricos, son muy pocos los estudios realizados sobre la recepción de la traducción en general, y menos los trabajos realizados desde un punto de vista experimental 
sobre un campo especializado de la traducción. Si bien numerosas teorías y estudios hablan del lector como pieza clave, muy pocos se centran en la recepción, y no existen apenas estudios empíricos sobre la recepción de textos traducidos.

Siguiendo esta misma idea, unos años más tarde, Mayoral (2005: 4) insiste en que como singularidad del estudio de la traducción audiovisual hay que señalar que en él la observación del producto (en particular la recepción del producto por el destinatario) puede arrojar mucha luz sobre el proceso. Este es otro, pues, de los argumentos para haber iniciado una línea de investigación sobre la recepción de la publicidad y las estrategias de traducción cuyos primeros resultados ofrezco en el presente trabajo.

\section{Aspectos metodológicos del estudio}

\subsection{Fuentes textuales}

He utilizado un corpus de textos audiovisuales de más de 800 espots televisivos. Se trata de anuncios grabados en los años 95-96, por una parte, y de anuncios grabados en los años 2000-2007, fundamentalmente de las cadenas Telecinco, Antena 3, Televisión Española, Televisión de Cataluña y Televisión Valenciana. También he utilizado para este trabajo los anuncios emitidos por el programa La rentadora (TV3), una selección excelente de los mejores anuncios de los 50 años de televisión en España. Finalmente, he contado asimismo con las selecciones de diversas ediciones de los Festivales de Publicidad de Cannes y de San Sebastián (2001-2006). Para la recopilación y gestión de la información he creado una base de datos en Access que cumple, aparte de objetivos investigadores, objetivos docentes, ya que la utilizo también para el análisis de anuncios en clases de traducción de la publicidad.

Como la figura de este apartado muestra (véase fig.1), la base de datos permite describir la información básica del producto: marca original, marca final, tipo de producto - accesorios y ropa, alimentación, bebidas alcohólicas, hogar, institucional, ocio, perfumes y cosmética, productos financieros, telecomunicaciones, otros productos -, destinatario - infantil, juvenil y adulto -, la agencia de publicidad que ha diseñado la campaña, el canal de donde hemos obtenido el espot y la fecha de emisión. Un segundo bloque de información permite realizar un análisis descriptivo del anuncio, siempre teniendo en cuenta el texto original y su traducción correspondiente para poder realizar análisis contrastivos. Así, se puede dar cuenta de aspectos profesionales cuando ha sido factible contactar con la agencia de publicidad o de traducción que ha realizado el trabajo; dejar constancia de la modalidad en que se traduce el artículo; analizar los diferentes códigos que concurren en el texto audiovisual original y traducido, siguiendo un esquema previamente establecido de los problemas que pueden presentar dichos 
textos y tratar los aspectos culturales relevantes y los casos de intertextualidad. Finalmente, he incorporado un bloque centrado en el texto y en el que presento la transcripción de los elementos linguísticos del texto original y del texto traducido y el propio texto audiovisual íntegro, bien a través de una conexión que hemos creado con la carpeta del corpus, bien a través de la conexión directa con una URL determinada.

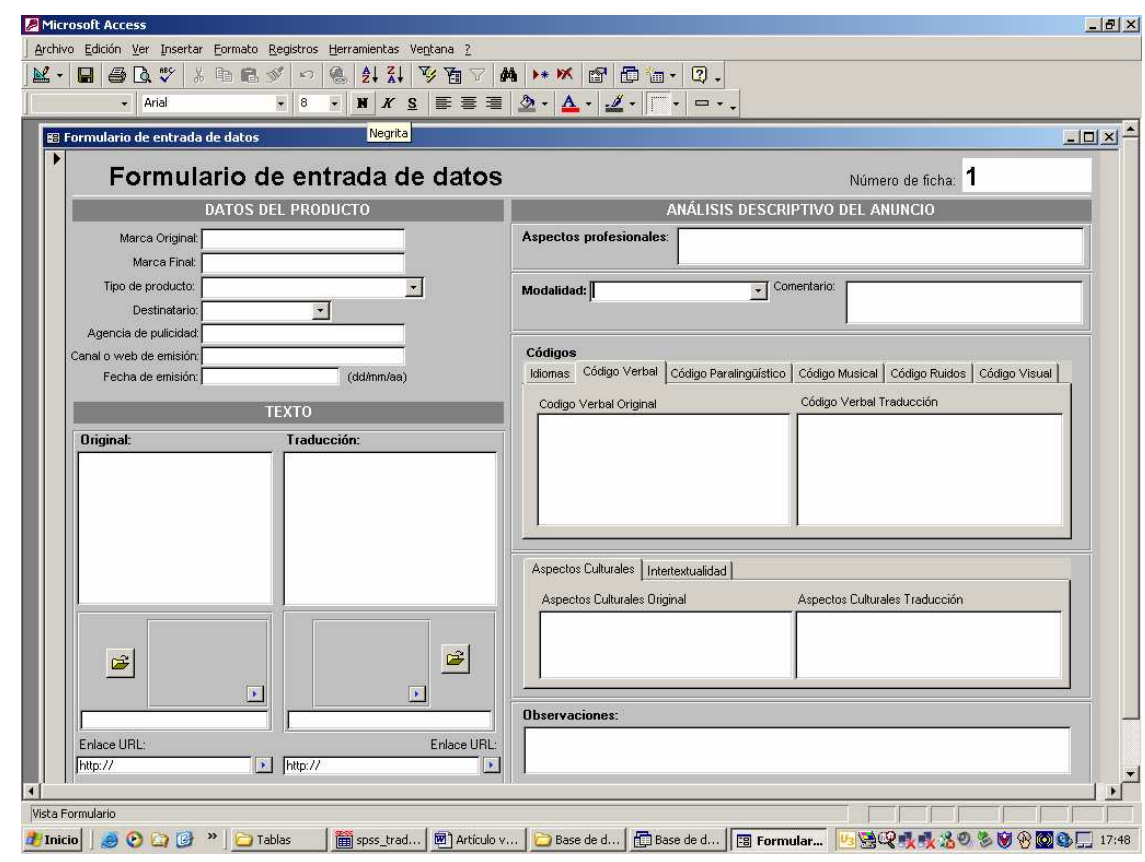

Figura 1. Formulario de entrada de datos

\subsection{Fuentes extratextuales}

Aparte de la consulta de la bibliografía básica sobre el tema, he llevado a cabo entrevistas con responsables de agencias de traducción, asesores lingüísticos de diferentes cadenas de televisión y responsables de agencias de traducción. Sin embargo, para este trabajo, he elaborado también una encuesta con la finalidad de obtener datos objetivos y fiables de los destinatarios de la traducción publicitaria televisiva para poder analizar la recepción de la misma y comprobar fehacientemente las hipótesis planteadas.

A pesar de que he tenido en cuenta trabajos de corte sociológico (León 1988; Monzó 2002), he adaptado algunas de las directrices a las necesidades del estudio. Por ejemplo, en cuanto al tipo de muestra, presento una combinación de encuesta de calle con encuesta controlada llevada a cabo en diferentes institutos públicos y privados y varios centros universitarios (estudiantes de Comunicación audiovisual, Publicidad y Relaciones públicas, Magisterio, Traducción e Ingeniería informática). 
He utilizado el programa SPSS (Statistical Product and Service Solutions) (Leechs 2005) para la recogida y el tratamiento de los datos. Se trata de un programa estadístico informático de uso habitual en las ciencias sociales y empresas de investigación de mercado. El programa permite trabajar con bases de datos de gran tamaño y se ajusta muy bien a las necesidades del usuario. Ofrece las posibilidades de dar formatos especiales a las salidas de los datos para su uso posterior (diagramas, tablas, gráficos); llevar a cabo pruebas exactas en muestras pequeñas, árboles de clasificación, etc.

La muestra tiene un carácter heterogéneo que no es en absoluto gratuito ya que he intentado obtener representación de distintos grupos de edad, sexo, nivel educativo, ámbito de estudios y distintos grados de conocimiento de idiomas.

Soy consciente de que los grupos poblacionales tienen frecuencias muy distintas (véase fig. 2 infra) pero considero muy interesante el hecho de dar más peso al segmento de población entre los 14 y los 25 años, ya que se trata de personas que han nacido y vivido en una España completamente democrática. Pensemos, por ejemplo, que hasta los años 80 , el idioma extranjero que predominaba como asignatura en la Educación General Básica era el francés. Esto cambia radicalmente a partir de los primeros años de aquella década, en los que el francés queda como asignatura prácticamente residual. Y el acceso al inglés en toda Europa en general ha permitido un avance de la globalización. El objetivo era obtener datos de grupos que han vivido situaciones educativas, políticas, sociales, etc. diversas e intentar prever algún cambio de tendencia en un futuro próximo.

\begin{tabular}{|c|r|r|r|r|}
\hline & Frecuencia & Porcentaje & Porcentaje válido & $\begin{array}{c}\text { Porcentaje } \\
\text { Acumulativo }\end{array}$ \\
\hline de 14 a 18 años & 196 & 42,6 & 42,6 & 42,6 \\
de 19 a 25 años & 156 & 33,9 & 33,9 & 76,5 \\
de 26 a 50 años & 65 & 14,1 & 14,1 & 90,7 \\
mayor de 50 años & 43 & 9,3 & 9,3 & 100,0 \\
Total & 460 & 100,0 & 100,0 & \\
\hline
\end{tabular}

Figura 2. Grupos poblacionales estudiados

La mayoría de las preguntas eran cerradas para facilitar el procesamiento de los datos. He dejado abiertas únicamente las preguntas referidas a si los encuestados recuerdan anuncios extranjeros subtitulados o en versión original para que especifiquen cuáles, en caso de una respuesta afirmativa.

A lo largo de este trabajo se considera como estadísticamente significativas las diferencias obtenidas cuando el valor de $p$ fue menor de 0,05 con las diferentes pruebas estadísticas, es decir, cuando la probabilidad de 
que las diferencias observadas se debieran al azar eran menores del 5\% $(p \leq 0,05)$.

En la figura 3, presentamos una muestra de la base de datos creada con el programa estadístico SPSS. En primer lugar tenemos una imagen de las variables utilizadas:

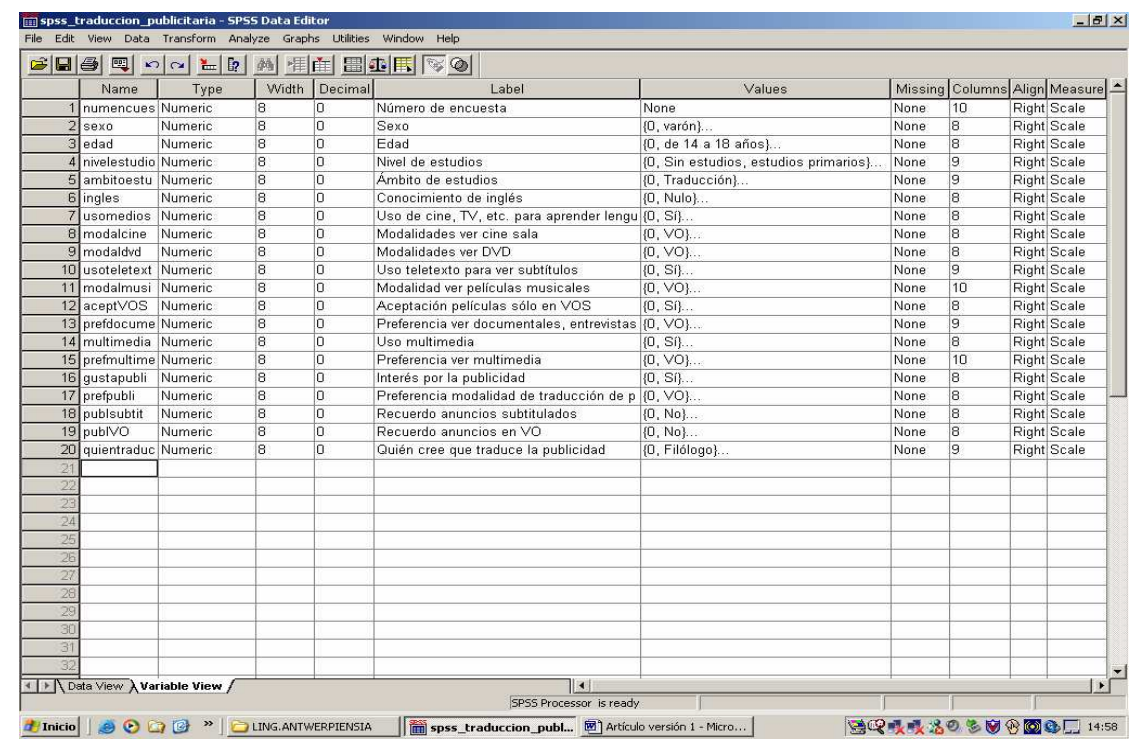

Figura 3. Variables de la encuesta

Aparte de los datos más personales, la encuesta constaba de 15 preguntas acerca de los usos y preferencias de los encuestados sobre cómo consumen los textos audiovisuales; la finalidad era conocer la relación de la población con las diferentes modalidades mayoritarias de traducción audiovisual, cuáles son sus gustos, preferencias, si son conscientes o no del papel del traductor, si conocen o reconocen su tarea; hemos querido establecer posibles relaciones entre la edad y el nivel de estudios de los encuestados y sus preferencias a la hora de consumir productos audiovisuales; su grado de aceptación de la publicidad traducida y la modalidad en que les gusta o les gustaría consumirla. Incluso he planteado un pequeño análisis prospectivo al añadir preguntas sobre las preferencias personales para ver si existen diferencias notables entre lo que los encuestados hacen (porque no tienen más remedio o porque es más fácil) y lo que les gustaría hacer. En la figura 4 se puede observar una muestra parcial de los datos obtenidos: 


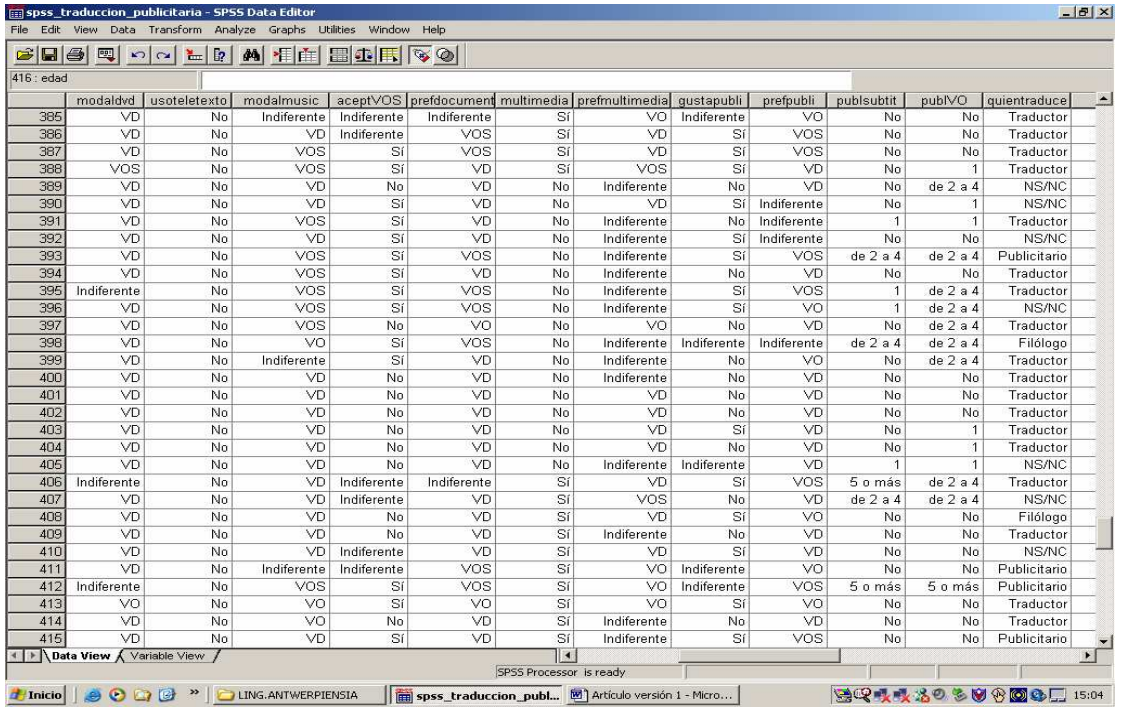

Figura 4. Muestra parcial de la base de datos

\section{Análisis de los datos}

Presento a continuación algunos de los datos que considero más relevantes del estudio que hemos realizado. Me gustaría de nuevo reiterar que, habiendo aplicado sistemáticamente la prueba del Chi-Square $\left(\chi^{2}\right)$, los resultados han sido siempre menores de 0.05 (de hecho, la inmensa mayoría eran del 0.001), lo cual demuestra que, en ningún caso, son fruto del azar. Así pues, podemos afirmar que el estudio resulta absolutamente fiable. Ofrezco los resultados siguiendo el orden de las hipótesis planteadas en el apartado 2 de este artículo.

\section{1. El doblaje es aún la modalidad más aceptada en la España actual}

En este apartado haremos alusión al consumo de doblaje y subtitulación en salas de exhibición. El $71 \%$ de los encuestados ve exclusivamente versiones dobladas. Tan solo un 2,4\% dice ver de forma habitual versiones originales (VO) y un $15,4 \%$ asistir a versiones originales subtituladas (VOS). Si buscamos una correlación con el nivel de estudios de cada encuestado, las personas sin estudios o con estudios primarios aceptan sólo en un 5,6\% la subtitulación, que asciende hasta el $70 \%$ en el caso de los licenciados. Atendiendo al conocimiento de inglés, aquellos que carecen de él aceptan exclusivamente el doblaje en un 96,8\%. La preferencia por la subtitulación aumenta a medida que los encuestados conocen mejor la lengua extranjera. Desde el punto de vista del ámbito de estudios de los encuestados, el consumo de subtitulación es mayor en los filólogos (25\%) y en los traductores $(12,7 \%)$ y desciende hasta el $12 \%$ en aquellos que no tienen formación en 
lenguas extranjeras. Otro factor que interviene pero que no he podido controlar en este trabajo es si el encuestado vive en ciudades o pueblos donde haya cines en VOS, haya filmotecas, amplia oferta cultural, etc. Nuestra intuición y experiencia nos dice que el consumo de versiones originales es mayor cuanto más próxima sea la oferta al lugar de residencia del espectador, y que las versiones originales se ofrecen mayoritariamente en núcleos urbanos importantes.

\subsection{Los espectadores consumen productos audiovisuales doblados por inercia y comodidad}

Elegir la opción del teletexto para ver los subtítulos y optar por el dual exige un esfuerzo extra al que muchos espectadores no estás habituados. Únicamente aquellos que buscan mejorar un nivel de lenguas suelen optar por el uso de las técnicas que están a nuestra disposición. Ello les permite acceder a la versión original a través del sistema dual y tener la ayuda de los subtítulos pensados, a priori, para la comunidad con dificultades auditivas, en el caso de que su conocimiento de la lengua extranjera no sea suficiente para poder comprender sin ayuda. Lo mismo ocurre con el DVD: en función del objetivo con el que los espectadores ven una película eligen una u otra opción. Esto se observa perfectamente al analizar los datos que nos proporciona la tabla comparativa entre el uso del teletexto para ver versiones originales por televisión con ayuda de la subtitulación y los diversos niveles de estudios: en general, los encuestados reconocen no utilizar el teletexto en un $87 \%$ que llega al $100 \%$ en el grupo de los que no tienen estudios o solo estudios primarios. Muchos de ellos afirman que, fundamentalmente, es por no hacer el esfuerzo y también por la costumbre e inercia de ver programación televisiva importada en versión doblada. Al introducir la variable "conocimiento de inglés", los resultados confirman que, aunque los encuestados son reacios a utilizar el teletexto, a medida que el dominio del idioma es mayor aumenta su uso. Aún así, el porcentaje en el grupo de conocimiento elevado del inglés llega únicamente al 21,6\%.

\subsection{La subtitulación comienza a ser más aceptada que antes por el pú- blico}

De hecho, resulta curioso que, cuando el público sólo tiene la posibilidad de ver las películas en VOS, como pueda ser el caso de Bowling for Columbine, de Michael Moore; La pasión de Cristo, de Mel Gibson, o Cartas desde Iwo Jima, de Clint Eastwood, por ejemplo, un 52\% de los encuestados aceptan la obligatoriedad de verlas subtituladas de muy buen grado. Es más, atendiendo a la variable de conocimiento de inglés, los resultados señalan que la aceptación de la VOS es del $19 \%$ entre los encuestados que no tienen ningún conocimiento de inglés y aumenta hasta el $74,2 \%$ en el segmento con un conocimiento elevado. 


\subsection{Los jóvenes tienen un conocimiento mayor de idiomas}

Hasta principios de los 90 la enseñanza obligatoria se denominaba Educación General Básica (EGB) y comprendía ocho cursos (6-13 años). Posteriormente, los jóvenes podían acceder a un trabajo, realizar una formación profesional (FP) o cursar el BUP (Bachillerato Unificado Polivalente, 14-16 años) y el COU (Curso de Orientación Universitaria, 17 años), tras el cual debían pasar una prueba para acceder a la universidad. El sistema actual de enseñanza en España es el siguiente: Educación infantil (3-5 años), optativa; Educación Primaria (6-11 años), obligatoria; Educación Secundaria obligatoria (12-15 años), conocida como ESO. Posteriormente, los jóvenes pueden incorporarse al mercado laboral, continuar los estudios a través de diferentes ciclos de formación o cursar el bachillerato (16-17 años), que les permite acceder a la universidad tras superar una prueba de selección que se lleva a cabo al final del segundo curso (PAU). El cambio de sistema educativo implica que, actualmente, todos los jóvenes cursan inglés de forma obligatoria hasta los 15 años. La tabla de la figura 5 es muy reveladora:

\begin{tabular}{|rr|r|r|r|r|r|}
\hline & \multicolumn{4}{|c|}{ Conocimiento de inglés } & \multirow{2}{*}{ Total } \\
\hline & & Nulo & Escaso & Aceptable & Elevado & \\
\hline Edad de 14 a 18 años & 2 & 43 & 139 & 12 & 196 \\
& & $1,0 \%$ & $21,9 \%$ & $70,9 \%$ & $6,1 \%$ & $100,0 \%$ \\
& de 19 a 25 años & 3 & 30 & 62 & 61 & 156 \\
& & $1,9 \%$ & $19,2 \%$ & $39,7 \%$ & $39,1 \%$ & $100,0 \%$ \\
& & 6 & 20 & 18 & 21 & 65 \\
& de 26 a 50 años & $9,2 \%$ & $30,8 \%$ & $27,7 \%$ & $32,3 \%$ & $100,0 \%$ \\
& & 20 & 14 & 6 & 3 & 43 \\
& mayor de 50 & $46,5 \%$ & $32,6 \%$ & $14,0 \%$ & $7,0 \%$ & $100,0 \%$ \\
& & 31 & 107 & 225 & 97 & 460 \\
& & $6,7 \%$ & $23,3 \%$ & $48,9 \%$ & $21,1 \%$ & $100,0 \%$ \\
\hline
\end{tabular}

Figura 5. Nivel de inglés por edades

Conviene especificar que los valores aplicados a la gradación de conocimientos siguen la clasificación propuesta por el CEFR (Common European Framework of Reference for Languages: Learning, Teaching, Assessment): escaso (A1); aceptable (B1); elevado (C1). Recordemos también que la mayoría de encuestados entre 14 y 18 años eran estudiantes de ESO y Bachillerato y que la mayoría de encuestados entre 19 y 25 años eran universitarios. 


\subsection{Los jóvenes se muestran más receptivos a los textos audiovisuales multilingües (VOS) en sus diferentes formatos}

Centrémonos, por ejemplo, en textos bien distintos: los documentales y entrevistas y los juegos de diversión multimedia. En cuanto a los primeros, prefieren la versión doblada el 50,5\% en el segmento entre 14 y 18 años, mientras que los mayores de 50 ya la prefieren en un $70 \%$. Los más interesados en las VOS son los segmentos que se encuentran entre los 19 y los 25 años $(53,8 \%)$ y de 26 a 50 años $(44,6 \%)$. Sobre los textos multimedia, mientras los jóvenes entre 14 y 18 años los consumen en un 82,7 \% y los prefieren en VOS en un 21,4\%, sólo los utilizan en un 2,3\% la población de más de 50 años y los prefieren en VOS únicamente el $2 \%$ de ellos.

\subsection{Cuanto mayor es el nivel de estudios de la población mayor es la aceptación de las versiones originales y subtituladas}

Los datos obtenidos han confirmado plenamente la hipótesis de partida. Así, por ejemplo, sobre la recepción de los musicales, el grupo sin estudios o estudios primarios las prefiere en un $11,1 \%$ en VOS, porcentaje que asciende hasta el $30,7 \%$ en el caso de los licenciados y hasta el $46,7 \%$ en el grupo de los doctores. En cuanto a los documentales y las entrevistas, el primer grupo los prefiere en VOS únicamente en un 5,6\%, mientras que destaca el $55,9 \%$ de preferencia de VOS por parte de los licenciados y el $66,7 \%$ en el segmento de doctores.

\subsection{A los consumidores les gusta la publicidad}

Con esta hipótesis se aborda ya el tema que nos atañe de forma más directa en este trabajo, pero que no puede entenderse sin el contexto de todo lo visto con anterioridad. Sobre los textos publicitarios me gustaría mostrar como punto de partida la siguiente frase: "Love them or hate them, you cannot avoid them" (Brierley 1995: 1, cit. en Valdés 2004: 17). Independientemente de la existencia de detractores y defensores de la publicidad, hay hechos innegables: la presencia e inversión en publicidad aumenta cada año. Su influencia es tal que muchos medios de comunicación de masas (prensa, radio y televisión) se financian, en gran parte, gracias a ella. Los datos del Boletín Mensual de TNS Audiencia de Medios de enero de 2007 muestran el índice de estacionalidad de la publicidad en España entre febrero de 2006 y enero de 2007 y presentan el número de anuncios emitidos por las televisiones, los minutos de pantalla y la inversión en miles de euros: los datos de febrero de 2006 indican que se emitieron 243.653 anuncios, con una duración total de 78.111 minutos y una inversión de 685.763 miles de euros; en los datos de enero de 2007 se aprecia un incremento de todos los valores (331.253 anuncios; 102.786 minutos y una inversión de 820.733 miles de euros). 
Los resultados de la encuesta acerca de la aceptación de la publicidad revelan que al $47 \%$ de los encuestados les gusta, al $32 \%$ no le gusta y al $20,9 \%$ le resulta indiferente.

\subsection{Los jóvenes prefieren la publicidad subtitulada}

Si escogemos el grupo de menor edad y el de mayor edad obtenemos los resultados siguientes: mientras un 17,3\% de los jóvenes prefiere la VO para la publicidad, el grupo de más de 50 años solo la admite en un 4,7\%; mientras un $27,6 \%$ del primer grupo opta por la VOS, el segundo lo hace en un $16,3 \%$; finalmente, mientras el $37,8 \%$ de los más jóvenes prefiere el doblaje en un $37,8 \%$, el grupo de mayores de 50 años lo eligen en un 53,5\%.

Resulta también muy significativo relacionar estos resultados con la variable de nivel de estudios ya que una buena parte de esos mayores son espectadores con estudios mínimos y la mayoría de los jóvenes entre 14 y 18 pertenecen al grupo de ESO. Así, es interesante comprobar cómo el grupo con estudios mínimos prefiere la VO en un $0 \%$, la VOS en un 5,6\% y el doblaje en un 94\%; sin embargo, el grupo de EGB, ESO elige la VO en un $21,1 \%$, la VOS en un $23,9 \%$ y el doblaje en un $42,3 \%$. Finalmente, el grupo de licenciatura opta por la VO en un $16,8 \%$, por la VOS en un 31,1 y la preferencia por el doblaje desciende hasta el $28,7 \%$.

\subsection{Los jóvenes recuerdan más que los mayores la publicidad original y la subtitulada}

El número de encuestados que recuerden muchos anuncios en otras lenguas distintas a las propias no es muy elevado. Uno de los factores es que tenían relativamente poco tiempo para responder. Concretamente, sobre los anuncios subtitulados, los mayores de 50 no recuerdan ningún anuncio en un $72,1 \%$ y no hay nadie capaz de recordar más de 5 . Sin embargo, como contrapunto, en el grupo de 19 a 25 años, el número de encuestados que no recuerda ninguno desciende a un $53,2 \%$ y aumenta a un $4 \%$ el grupo de los que son capaces de recordar más de 5 . Como dato interesante hay que destacar que los productos más nombrados son los coches de bajo coste y la ropa deportiva, que coincide con un destinatario joven. En cuanto a la publicidad emitida exclusivamente en versión original, las diferencias no son tan notables; de hecho, hay una aparente contradicción respecto a los datos anteriores: el grupo entre 14 y 18 años no recuerda ningún anuncio en un $73 \%$, mientras que los mayores de 50 años sólo en un $65,1 \%$. Merece destacarse que los productos más citados fueron los perfumes, cuyo target (segmento de población considerado como consumidor potencial prototípico) coincide mayoritariamente con el público adulto y con poder adquisitivo.

Respecto a los resultados de la encuesta llevada a cabo en este estudio, queremos destacar que los encuestados recuerdan, mayoritariamente, anuncios subtitulados de coches y bebidas y anuncios en versión original de 
perfumes (francés, primero; pero últimamente también los recuerdan en inglés). Merece destacarse el posicionamiento del anuncio de BMW que ha tenido como personaje central a Bruce Lee, con un eslogan que da lugar a toda una cadena de intertextos (Be water, my friend). Según TsnNews (2007: 5), este tipo de campañas aumentan entre un 15 y un $30 \%$ la percepción de la marca. Aunque el anuncio estaba subtitulado, resulta altamente significativo que la totalidad de las personas encuestadas que lo recordaban, en su mayoría jóvenes, lo hacían no en español (Sé agua, amigo mío) o en las diferentes lenguas autonómicas, sino en inglés. Este anuncio subtitulado ha servido incluso como punto de partida para otros anuncios de empresas de automoción distintas (Mitsubishi, noviembre de 2006): así, se pasa del eslogan No te adaptes a la carretera, sé la carretera de BMW a No te adaptes al campo. Sé de campo para anunciar un todoterreno. Incluso ha sido objeto de numerosas parodias en programas de humor de televisiones de España, tanto de ámbito estatal como autonómico. Una muestra más del poder e importancia de la publicidad.

5.10. Los encuestados consideran que la traducción publicitaria la llevan a cabo profesionales de la traducción frente a profesionales de la publicidad o lingüistas

A partir de mi experiencia como profesora de traducción de la publicidad en la licenciatura de Publicidad y Relaciones Públicas y de la lectura de algunos expertos en el tema (Sabaté 1999), diseñé cuatro respuestas posibles para la pregunta "Quién cree que traduce la publicidad". Así, los encuestados podían responder que la publicidad la traducen especialistas en lengua (filólogos); especialistas en publicidad (publicitarios) o especialistas en traducción (traductores). Incluí también la opción de no sabe/no contesta para observar de cara a otros trabajos futuros si el grado de desconocimiento o de falta de reflexión sobre el tema era o no significativo. Los datos obtenidos aparecen en la figura 7: 


\begin{tabular}{|ll|r|r|r|r|r|}
\hline & \multicolumn{4}{|c|}{ Quién cree que traduce la publicidad } & Total \\
\hline & & Filólogo & Traductor & Publicitario & NS/NC & \\
\hline $\begin{array}{l}\text { Ámbito de } \\
\text { estudios }\end{array}$ & Traducción & 3 & 49 & 24 & 30 & 106 \\
& & $2,8 \%$ & $46,2 \%$ & $22,6 \%$ & $28,3 \%$ & $100,0 \%$ \\
& Filología & 3 & 6 & 6 & 5 & 20 \\
& & $15,0 \%$ & $30,0 \%$ & $30,0 \%$ & $25,0 \%$ & $100,0 \%$ \\
& Estudios & 2 & 53 & 19 & 16 & 90 \\
& afines & $2,2 \%$ & $58,9 \%$ & $21,1 \%$ & $17,8 \%$ & $100,0 \%$ \\
& & 7 & 136 & 52 & 49 & 244 \\
& No relación & $2,9 \%$ & $55,7 \%$ & $21,3 \%$ & $20,1 \%$ & $100,0 \%$ \\
& & 15 & 244 & 101 & 100 & 460 \\
& & $3,3 \%$ & $53,0 \%$ & $22,0 \%$ & $21,7 \%$ & $100,0 \%$ \\
\hline
\end{tabular}

Figura 7. Quién cree que traduce la publicidad

Si utilizamos la variable "nivel de estudios" los resultados muestran que cuanto menor es el nivel de estudios mayor es la creencia de que es el traductor el encargado de traducir estos textos $(77,8 \%$ para los traductores frente al 16,7\% para los publicitarios); y que cuanto mayor es el nivel de estudios mayor es la creencia de que son los publicitarios quienes se encargan de ello (46,7\% para los publicitarios frente al $26,7 \%$ para los traductores).

\subsection{Existe una relación muy estrecha entre el tipo de producto y des- tinatario de un anuncio, por una parte, y la estrategia de traduc- ción elegida}

En la base de datos en Access creada, los datos obtenidos señalan, por ejemplo, que cerca del $40 \%$ de los espots de perfumes se emiten en versión original o versión subtitulada, y van dirigidos en su mayoría a un público adulto cuando el producto es francés (Dior, Cacharel, Lancôme, etc.) y más joven cuando el producto es de origen norteamericano (Hugo Boss, Hilfiger, etc.). Los anuncios de coches de lujo dirigidos a un público adulto y de alto poder adquisitivo se doblan (Mercedes, Audi), mientras que algunos anuncios de coches dirigidos a un sector más joven, aparecen ya subtitulados (Seat Altea, Smart con Robbie Williams cantando Feel).

\subsection{La globalización aumenta la necesidad de diseñar publicidad "ap- ta para todos los públicos"}

Así, hay determinados grupos de productos que son más susceptibles de adoptar estrategias de traducción más extranjerizantes (no traducción y subtitulación). De la base de datos se desprende que en este grupo de estrate- 
gias más extranjerizantes predominan el grupo de bebidas alcohólicas y refrescos, cosmética y perfumes, coches de bajo coste, mientras que los productos más relacionados con la idiosincrasia de cada país optan por el doblaje o la creación de espots ad hoc (hogar, alimentación y productos financieros, entre otros).

Por ejemplo, respecto a los anuncios de productos deportivos o que utilizan como leitmotiv el deporte, se observa el paso de una tendencia más estandarizadora u otra más global: a finales de los 80, McDonalds utilizó a dos estrellas del baloncesto de la NBA (Larry Bird y Michael Jordan) para su campaña y todo estaba doblado sistemáticamente. En los 90, Nike con su eslogan Just do it comienza a emitir en España y otros países europeos una serie de anuncios con jugadores internacionales de fútbol de gran prestigio (Figo, Ronaldo, Cantona, etc.) y elige la modalidad de subtitulación. Esta tendencia continúa en los años 2000 junto con campañas en las que los eslóganes permanecen invariablemente en inglés, aunque los textos se doblan unas veces (Adidas con su Impossible is nothing y las campañas dobladas con Mohammed Ali como protagonista), se subtitulan otras (las de Adidas con Nadia Comaneci) o se presentan en versión original exclusivamente, como las de Nike con su A little less hurt y la canción Hurt, de Johnny Flow, toda una muestra de intertextualidad.

\section{La sociedad española y el multilingüismo}

Con la globalización, las campañas publicitarias han experimentado cambios notables ya que se diseñan pensando en el mayor número posible de destinatarios. La alianza entre las grandes multinacionales y los medios de comunicación de masas resulta de lo más productiva: la televisión digital, los canales internacionales, Internet, etc. Como señala Bueno (2000: 117) no hay un comportamiento homogéneo a la hora de decidir una estrategia de diseño y de traducción de la campaña, pero sí que es cierto que existen tendencias, aunque lo cierto es que en España se detecta una ambivalencia constante. Sin embargo, parece que, cada vez más, y debido a factores como la homogeneización de los mercados y las políticas económicas, la creación de espacios comunes y una cierta estandarización de los hábitos de consumo, las agencias van aumentando la tendencia de diseñar campañas comunes.

Llegados a este punto, me parece muy interesante hacer referencia a toda una serie de trabajos que, hechos desde la perspectiva de la publicidad tratan también, de forma más o menos explícita, según los casos, sobre la traducción de estos textos. Se trata de estudios que introducen la ideología como un factor central que condiciona la forma en que se elabora y se traduce la publicidad; que analizan los conceptos de mundialización, (surgido a finales del siglo XIX y relacionado con la idea de solidaridad), de internacionalización (anglicismo adoptado por las fuerzas políticas y económicas del siglo XX) y de globalización (que procede del mundo financie- 
ro y que surge a finales del siglo XX), para analizarlos de forma crítica y profundizar en los problemas que plantea la circularidad global/local. Mattelart (1999 y 2006) es uno de los autores que pone en tela de juicio consignas dominantes hasta hace poco como la idea de que la publicidad ha de ser lo más global posible, con lo cual las campañas ideales serían aquellas que no necesitan de la traducción. Sin embargo, en los últimos años, ha irrumpido con fuerza el concepto de publicidad glocal, término en el que confluyen las palabras global y local, que marca la mayor parte de la publicidad actual. Valdés (2004) comenta al respecto que la publicidad ha ido pasando de la consigna Think global, act local resumida en el neologismo visto con anterioridad, en el que hay un diseño y objetivos globales para la campaña y una adaptación posterior a cada mercado concreto, al Think local, act global. Esta última tendencia ha sido posible gracias a los medios de comunicación de masas como la televisión o la red.

Pereira (2001: 9) afirma que la sociedad española actual se muestra receptiva y abierta hacia el cambio hacia el consumo cada vez más habitual del subtitulado y lo relaciona directamente con "el deseo de conocer otras culturas y aprender otras lenguas distintas a la suya" ( $c f$. el Eurobarómetro especial 243 "Los europeos y sus lenguas" 2006).

A este respecto, Valdés (2004: 93-94) señala que la circunstancia de que "los jóvenes españoles posean un conocimiento mayor de idiomas también ha influido en el uso de lenguas extranjeras en los anuncios, como demuestra el gran número de anuncios no traducidos del inglés que llenan las páginas de las publicaciones españolas cuyo público receptor pertenece al sector joven de la población.

En este sentido, hay que agradecer algunas campañas hechas desde determinadas instituciones para promocionar el consumo de versiones originales mediante el uso del sistema dual y las versiones subtituladas del teletexto para fomentar el conocimiento del inglés y de otras lenguas. Tal ha sido el caso de la Televisió de Catalunya que, durante todo el mes de marzo de 2007 preparó dentro de su programación de continuidad la campaña sobre Innovación L'anglès a casa ("El inglés en casa"). A pesar de la conveniencia de que se empiece a promocionar el multilingüismo desde la televisión, la campaña parte de un punto erróneo: la confusión entre la subtitulación para sordos y la subtitulación destinada a personas sin problemas auditivos. Se trata de trasvases que tienen características diferentes y que, vistos por un público que no es el destinatario real, pueden crear sensación de redundancia o de falta de información, según el caso.

\section{Conclusiones}

Recién estrenado el siglo XXI, Pereira (2001: 8-9) comentaba que la consideración social del subtitulado en España estaba experimentando cambios sustanciales: 
Aunque hay autores (Luyken, 1991) que consideran tarea inútil y del todo imposible intentar cambiar las preferencias del público hacia una u otra modalidad de traducción audiovisual distinta de aquella a la que está acostumbrado, un análisis minucioso de la oferta audiovisual española basta para rebatir esta tajante afirmación. (ibid.: 8)

Actualmente, hay toda una serie de intersticios entre los cuales va introduciéndose, poco a poco, una modalidad considerada hasta hace poco muy elitista por estar íntima y casi exclusivamente relacionada con el denominado cine de arte y ensayo.

Del análisis del extenso corpus de anuncios se desprenden, entre otros, los siguientes datos y consideraciones: en las primeras décadas de existencia de la televisión española, la publicidad era un fiel reflejo de la sociedad autárquica del franquismo con un uso de productos nacionales en su inmensa mayoría y uso exclusivo del español por imperativo legal. En este sentido, coincido plenamente con lo expuesto por Menor Sendra (2006: 76) cuando describe la televisión durante la dictadura franquista:

La difusión era exclusivamente en castellano. No se permitían otras lenguas españolas e incluso las producciones audiovisuales de otros países debían doblarse al castellano. Franco quería que la televisión y el sistema educativo completasen la tarea de uniformización lingüística que los franceses acometieron varias generaciones atrás y en la que los liberales españoles habían fracasado.

A medida que el país se abre a Europa y el mundo, allá por los últimos años de la década de los 60, irrumpen en los hogares españoles una gran cantidad de productos extranjeros cuyos anuncios están hechos mayoritariamente en España o bien doblados al español. El final de los años 80 y el principio de los años 90, supone la aparición en el mundo de la publicidad de otras lenguas distintas a la española: el mundo de la cosmética y la moda, como el anuncio de Trésor de Lancôme (Le parfum des instants précieux), o el famoso Lou Lou C'est moi, de la firma Cacharel, nos descubren el francés asociado al mundo del glamour y la sofisticación (el buen perfume es francés y la presencia del idioma pretende reforzar esta idea); algunos anuncios de pasta ya utilizan el italiano como factor que ayuda al posicionamiento de marcas determinadas (Buitoni) y las canciones en inglés son algo ya muy habitual en una gran cantidad de anuncios. El multilingüismo parece consustancial al multiculturalismo y a la globalización. Ya a partir del 2000, aparecen de forma un tanto más habitual una serie de campañas destinadas a un público joven en los que la modalidad elegida para el transvase lingüístico es la subtitulación porque permite dar una imagen de modernidad, internacionalización e identidad juvenil mucho mayor: el inglés hace acto de presencia en anuncios de cerveza (Budweiser: Whassup?), refrescos (Pepsi: Ask for more), artículos deportivos (Nike: Just do it; Adidas: Impossible is nothing), coches de bajo coste (Seat Leon), productos presentados 
por personajes famosos (Georges Clooney en Nespresso: What else?, de Nestlé; Madonna en moda H\&M: This, this; Bruce Lee en BMW: "Be water, my friend", John McEnroe It's in! en Seat Altea, Jay-Z en Reebok: I am what I am, Juliette Binoche en perfumes de Lancôme: Poême, etc.), productos de cosmética destinados a este segmento de población (colonias de Hugo Boss: Fragances for men; Dolce\&Gabana: The one; Dior: C'est ça que J'adore), etc.

Por otra parte, considero que actualmente y en un futuro a corto y medio plazo hay toda una serie de factores que pueden incidir en la aceptación de la subtitulación por parte de los telespectadores españoles: Internet también ha influido en la mayor tolerancia del público joven respecto a la subtitulación ya que fenómenos como el de la página web youtube.com en el que aparecen una gran cantidad de textos subtitulados han ido familiarizando a un segmento amplio de la sociedad española actual, fundamentalmente los jóvenes entre 14 y 25 años, con los subtítulos; los programas de edición de subtítulos que se utilizan en una gran cantidad de hogares españoles en tareas tan cotidianas como la creación de películas caseras ha puesto en contacto directo al espectador con una técnica de la cual se desconocía casi todo. Finalmente, la nueva Ley General Audiovisual, actualmente en fase parlamentaria, está posibilitando que, poco a poco, la subtitulación para sordos haga acto de presencia en nuestras casas cada día. Pensemos que en 2015 toda la televisión deberá cumplir las normas de accesibilidad (Pereira 2005, Orero 2006). Actualmente, accedemos a ella a través del teletexto y el sistema dual para personas con deficiencias visuales, con la audiodescripción, pero ya hay muchos organismos que presentan las campañas institucionales de forma accesible y directa. Entendemos que en un plazo muy breve ya podremos estudiar objetivamente la relación entre la subtitulación para sordos y la mayor aceptación de la subtitulación en general.

\section{Bibliografía}

Adab, Beverly \& Cristina Valdés (eds) (2004). "Key Debates in the Translation of Advertising Material". Special Issue The Translator, 10(2). Manchester: Saint Jerome.

Bueno, Antonio (2000). Publicidad y Traducción. Monográficos de la Revista Hermeneus, vol.2. Soria: Diputación de Soria.

Bueno, Antonio (2005). "La didáctica de la traducción de textos publicitarios", en Zabalbeascoa, Patrick et al. (eds). La traducción audiovisual. Investigación, enseñanza y profesión. Granada: Comares, 231-240.

Comisión Europea (2006). Eurobarómetro especial 243 "Los europeos y sus lenguas". http://ec.europa.eu/public_opinion/archives/ebs/ebs_243_sum_es.pdf. (consulta a 27 de noviembre de 2007).

DD.AA. 2007. Tns News. $\mathrm{n}^{\circ}$ 28. Invierno/primavera.

DD.AA. 2007. Boletín Mensual de TNS Audiencia de Medios, enero 2007.

Fuentes Luque, Adrián (2001). "Estudio empírico sobre la recepción del humor audiovisual", en Lorenzo, Lourdes \& Ana María Pereira (eds). Traducción subordinada (II). El subtitulado (inglés-español/galego). Vigo: Servicio de Publicacións Universidade de Vigo, 69-84.

Guidère, Mathieu (2000). Publicité et traduction. París: Harmattan.

Leech, Nancy L. et al. (eds) (2005). SPSS for Intermediate Statistics: Use and Interpretation. London/Mahmway, New Jersey: Lawrence Erlbaum Associates, Publishers. 
León, José Luis (1988). La investigación en publicidad. Metodologías y crítica. Bilbao: Servicio editorial Universidad del País Vasco.

Mattelart, Armand (1990). La internacional publicitaria. Madrid: Fundación para el Desarrollo de la Función Social de las Comunicaciones.

Mattelart, Armand. (2006). Diversidad cultural y mundialización. Barcelona: Paidós Comunicación.

Mayoral, Roberto (2005). "Reflexiones sobre la investigación en traducción audiovisual", en Zabalbeascoa, Patrick et al. (eds). La traducción audiovisual. Investigación, enseñanza y profesión. Granada: Comares, 3-8.

Menor Sendra, Juan 2006. "El fracaso de la nacionalización mediática: el ejemplo de la televisión del franquismo", en Pérez-Amat, Ricardo et al. (eds). Sociedad, integración y televisión en España. Madrid: Ediciones del Laberinto, 41-108.

Monzó, Esther (2002). La professió del traductor jurídic i jurat: descripció sociològica del profesional i anàlisi discursiva del transgènere. Castelló de la Plana. Tesis doctoral.

Munday, Jeremy (2004). "Advertising: Some Challenges to Translation Theory", en Adab, Beverly \& Cristina Valdés (eds) (2004). Key Debates in the Translation of Advertising Material. Special Issue The Translator, vol.10:2. Manchester: Saint Jerome, 199-219.

Orero, Pilar (2006). "Algunas consideraciones sobre la audiodescripción comercial", en PérezAmat, Ricardo et al. (eds). Sociedad, integración y televisión en España. Madrid: Ediciones del Laberinto, 277-292.

Pereira, Ana María (2001). "Subtitulado y traducción en España y en Galicia: su historia”, en Lorenzo, Lourdes \& Ana María Pereira (eds). Traducción subordinada (II). El subtitulado (inglés-español/galego). Vigo: Servicio de Publicacións Universidade de Vigo, 5-10.

Pereira, Ana María (2005). "El subtitulado para sordos: estado de la cuestión en España, Quaderns, $12,161-172$.

Rodríguez del Bosque, Ignacio et al. (eds) (1997). Comunicación comercial: conceptos y aplicaciones. Madrid: Editorial Civitas, S.A.

Séguinot, Candace (1994). "Translation and Advertising: Going Global". Current Issues in Language and Society 1 (3), 249-265.

Sabaté, Joan (1999). La publicitat en català. Barcelona: Pòrtic. Centre d'Investigació de la Comunicació.

Snell-Hornby, Mary (2006). The Turns of Translation Studies. Amsterdam: John Benjamins.

Valdés, Cristina (2000). "Reception Factors in Multimedia Translation: The Case of Advertisements", en Chesterman, Andrew, Natividad Gallardo \& Yves Gambier (eds) Translation in Context. Selected Contributions from the EST Congress, Granada 1998. Amsterdam/Philadelphia: John Benjamins, 271-280.

Valdés, Cristina (2001). "Extranjerización y adaptación en la traducción de espots publicitarios", en Agost, Rosa y Frederic Chaume (eds). La traducción en los medios audiovisuales. Castelló: Servei de Publicacions de la Universitat Jaume I, 183-192.

Valdés, Cristina (2004). La traducción publicitaria: comunicación y cultura. Bellaterra; Castelló de la Plana; Barcelona; València: UAB; UJI; UPF; UV.

Valdés, Cristina (2005). "Metodología y propuesta de un modelo teórico para el estudio de la traducción de textos publicitarios", en Zabalbeascoa, Patrick et al. (eds). La traducción audiovisual. Investigación, enseñanza y profesión. Granada: Comares, 153-163.

\section{Filmografia}

Bowling for Columbine (2002)

Canadá, Estados Unidos, Alemania.

Dir. Michael Moore

La pasión de Cristo (2004)

Estados Unidos.

Dir. Mel Gibson
Cartas desde Iwo Jima (2006)

Estados Unidos, Japón.

Dir. Clint Eastwood 
${ }^{1}$ Queremos agradecer la colaboración de David Martínez en la confección de la base de datos SPSS; a Víctor González, la aportación al diseño de la base de datos en Access; a Vicente Traver, Enriqueta Planelles, Nicolás Joaquín Blas, Esther Monzó, Justine Brehm, Natividad Juste, Carmen Peña y Glòria Torralba, su ayuda a la hora de pasar las encuestas en institutos y centros universitarios. 